\title{
Erratum
}

\section{In the article}

\section{«Reproductive Immunology - an Update»}

(Transfus Med Hemother 2006;33:474-485) one author was accidentally left out.

The full author list must read:

Tobias G. Poehlmann ${ }^{\mathrm{a}} \quad$ Justine S. Fitzgerald ${ }^{\mathrm{a}}$ Susann Busch ${ }^{\mathrm{a}} \quad$ Ekkehard Schleussner $^{\mathrm{a}}$ Gabriela Gutiérrez ${ }^{a, b}$ Sandra Blois ${ }^{c}$ Petra C. Arck ${ }^{c}$ Sabine Engert ${ }^{d} \quad$ Ulrike Kämmerer $^{d}$ Julia Szekeres-Bartho ${ }^{\mathrm{e}}$ Udo R. Markert ${ }^{\mathrm{a}}$

a Placenta-Labor, Abteilung für Geburtshilfe, Friedrich-Schiller-Universität Jena, Germany

bInmunología, Facultad de Farmacia y Bioquímica, Universidad de Buenos Aires / IDEHU, Argentina

c PsychoNeurolmmunologisches Labor, Biomedizinisches Forschungszentrum Charité, Campus Virchow, Universitätsmedizin Berlin,

${ }^{\mathrm{d}}$ Frauenklinik, Universität Würzburg, Germany

${ }^{e}$ Reproductive and Tumor Immunology, Research Group of the Hungarian Academy of Sciences, Department of Medical Microbiology and Immunology, Pecs University, Medical School, Pecs, Hungary

We apologize for this mistake. 